\title{
Universality of the EMC effect
}

\author{
Jiunn-Wei Chen ${ }^{\mathrm{a}, \mathrm{b}}$, William Detmold ${ }^{\mathrm{c}}$ \\ a Department of Physics and National Center for Theoretical Sciences at Taipei, National Taiwan University, Taipei 10617, Taiwan \\ b Lawrence-Berkeley Laboratory, Berkeley, CA 94720, USA \\ ${ }^{c}$ Department of Physics, University of Washington, Seattle, WA 98195-1560, USA
}

Received 2 August 2005; accepted 11 August 2005

Available online 24 August 2005

Editor: L. Alvarez-Gaumé

\begin{abstract}
Using effective field theory, we investigate nuclear modification of nucleon parton distributions (for example, the EMC effect). We show that the universality of the shape distortion in nuclear parton distributions (the factorisation of the Bjorken $x$ and atomic number $(A)$ dependence) is model independent and emerges naturally in effective field theory. We then extend our analysis to study the analogous nuclear modifications in isospin and spin-dependent parton distributions and generalised parton distributions.
\end{abstract}

() 2005 Elsevier B.V. All rights reserved.

In 1983, the European Muon Collaboration (EMC) reported measurements [1] of the ratio $R_{\mathrm{Fe}}(x)$ of $F_{2}(x)$ structure functions in iron and deuterium in deep inelastic scattering (DIS). In the parton model, these structure functions are defined as $F_{2}^{A}(x)=\sum_{i} Q_{i}^{2} x \times$ $\left[q_{i}^{A}(x)+\bar{q}_{i}^{A}(x)\right]$, where $q_{i}^{A}(x)\left[\bar{q}_{i}^{A}(x)\right]$ is the parton distribution function (PDF) for quarks (anti-quarks) of flavor $i$ in a nucleus, $A$, and $Q_{i}$ is the electric charge of $q_{i}$. Bjorken $x$ is the longitudinal momentum fraction of the struck parton in the infinite momentum frame with respect to its parent nucleon. $R_{\mathrm{Fe}}(x)$ is normalised to unity if the nucleons in the nuclei are

E-mail addresses: jwc@ @hys.ntu.edu.tw (J.-W. Chen), wdetmold@phys.washington.edu (W. Detmold). non-interacting. The main observation of EMC was that $R_{\mathrm{Fe}}(x)$ deviated from unity by up to $20 \%$ over the range $0.05<x<0.65$ in which measurements were taken. The EMC result was unlooked for and came as a surprise to many physicists at the time partly because the typical binding energy per nucleon is so much smaller $(<1 \%)$ than the nucleon mass and the energy transfer involved in a DIS process.

Over the past two decades, further experiments have been performed by many groups (see Refs. [2-5] for recent reviews) aiming to better understand the details of nuclear modifications of hadron structure functions (here referred to generically as the EMC effect). The EMC result has been confirmed and demonstrated in many other nuclei ranging from helium to lead. Nuclear modification of structure func- 
tions has also been studied in other situations such as proton-nucleus Drell-Yan experiments and quarkonium production $[5,6]$. Overall, a very interesting picture has emerged; for an isoscalar nucleus of atomic number $A$, the shape of the deviation from unity of $R_{A}(x)=F_{2}^{A}(x) / A F_{2}^{N}(x)$ (here $F_{2}^{d}$ has been converted to the isoscalar $F_{2}^{N}$ subject to a small model dependent error, and the slight $Q^{2}$ dependence of $R_{A}(x)$ [5] is suppressed) is universal [7,8], namely independent of $A$ within experimental error bars, while the magnitude of the distortion is empirically proportional to the number density of the nucleus, $\rho_{A}$ [9]. Fits to the available data that support these features will be presented elsewhere [10]. These findings have inspired many theoretical analyses seeking to understand the details of the EMC effect in various approaches (see the reviews for summaries). Different physical processes have been identified as the causes of the modifications in different $x$ regions; e.g., nuclear shadowing at low $x$ and Fermi motion at large $x$. A recent conclusion of detailed model studies was that the EMC effect necessarily implies modification of the nucleon PDFs and cannot be explained through traditional nuclear physics [11]. However, little is known directly from QCD.

In this Letter, we employ effective field theory (EFT) to investigate the EMC effect by studying nuclear matrix elements of the twist-two operators which are related to parton distributions and structure functions via the operator product expansion. We find that the universality of the shape distortion of the EMC effect is a model independent result, arising from the symmetries of QCD and the separation of the relevant scales. The $x$ dependence of $R_{A}(x)$ is governed by short distance physics, while the overall magnitude (the $A$ dependence) of the EMC effect is governed by long distance matrix elements calculable using traditional nuclear physics. We then proceed to study analogous nuclear effects in isospin-odd, and spindependent parton distributions and generalised parton distributions (GPDs) [12]. We also discuss aspects of extracting the shape of the EMC effect from first principles using lattice QCD. This approach provides a clear connection between the EMC effect and many other observations of nuclear modification of hadron properties.

EFT is a model independent approach which only makes use of the symmetries and scale separation of the system (for recent reviews see Ref. [13]). This approach has been successfully applied to many low energy processes in $A=1,2,3,4$ systems. Recently EFT has been applied to the computation of hadronic matrix elements of twist-two operators in the meson and single nucleon sectors [14-16] and applied to chiral extrapolations of lattice calculations of moments of parton distributions [17]. The approach has also been extended to analyse moments of generalised parton distributions [18], large $N_{C}$ relations among PDFs in nucleons and the $\Delta$-isobar [15], and deeply virtual Compton scattering in the nucleon [19] and deuteron systems [20]. The method is readily generalised to the multi-nucleon case. Although we will concentrate on quark bilinear twist-two operators, the framework can be easily applied to gluonic operators and thereby to nuclear effects in gluonic distributions which are important in heavy ion collisions at RHIC and LHC and at a future Electron-Ion Collider.

To described the EMC effect observed in $F_{2}$ data on isoscalar nuclei, we consider the normalised, spin singlet, isoscalar twist-two operators,

$\mathcal{O}_{q}^{\mu_{0} \cdots \mu_{n}}=\bar{q} \gamma^{\left(\mu_{0}\right.} i D^{\mu_{1}} \ldots i D^{\left.\mu_{n}\right)} q /\left(2 M^{n+1}\right)$,

where $(\cdots)$ indicates that enclosed indices have been symmetrised and made traceless, $D^{\mu}=\left(\vec{D}^{\mu}-\overleftarrow{D}^{\mu}\right) / 2$ is the covariant derivative and $M$ is the nucleon mass. The matrix elements of $\mathcal{O}_{q}^{\mu_{0} \cdots \mu_{n}}$ in an unpolarised single nucleon state with momentum $P$ can be parametrised as

$\left\langle P\left|\mathcal{O}_{q}^{\mu_{0} \cdots \mu_{n}}\right| P\right\rangle=\left\langle x^{n}\right\rangle_{q} \tilde{v}^{\mu_{0}} \cdots \tilde{v}^{\mu_{n}}$,

where the nucleon velocity $\tilde{v}^{\mu}=P^{\mu} / M$. It is well known that the coefficients $\left\langle x^{n}\right\rangle_{q}$ correspond to moments of the isoscalar combination of parton distribution functions,

$\left\langle x^{n}\right\rangle_{q}=\int_{-1}^{1} d x x^{n} q(x)$,

where $q(x)$ is the isoscalar quark distribution and $q(-x)=-\bar{q}(x)$.

We first consider only nucleonic degrees of freedom (i.e., assume that pions are integrated out of the EFT - they will be reintroduced below) and perform the standard matching procedure in EFT, equating the quark level twist-two operators to the most general combinations of hadronic operators of the same 
symmetries [14-17]. The leading one- and two-body hadronic operators in the matching are

$$
\mathcal{O}_{q}^{\mu_{0} \cdots \mu_{n}}=\left\langle x^{n}\right\rangle_{q} v^{\mu_{0}} \cdots v^{\mu_{n}} N^{\dagger} N\left[1+\alpha_{n} N^{\dagger} N\right]+\cdots
$$

where $v^{\mu}=\tilde{v}^{\mu}+\mathcal{O}(1 / M)$ is the velocity of the nucleus. Operators involving additional derivatives are suppressed by powers of $M$ in the EFT powercounting. In Eq. (4) we have only kept the SU(4) (spin and isospin) singlet two-body operator $\alpha_{n} v^{\mu_{0}} \ldots \times$ $v^{\mu_{n}}\left(N^{\dagger} N\right)^{2}$. The other independent two-body operator $\beta_{n} v^{\mu_{0}} \cdots v^{\mu_{n}}\left(N^{\dagger} \tau N\right)^{2}$, which is non-singlet in $\mathrm{SU}(4)$ ( $\boldsymbol{\tau}$ is an isospin matrix), is neglected because $\beta_{n} / \alpha_{n}=O\left(1 / N_{c}^{2}\right) \simeq 0.1$ [21], where $N_{c}$ is the number of colors. Furthermore, the matrix element of $\left(N^{\dagger} \tau N\right)^{2}$ for an isoscalar state with atomic number $A$ is smaller than that of $\left(N^{\dagger} N\right)^{2}$ by a factor $A$ [10]. Three- and higher-body operators also appear in Eq. (4); numerical evidence from other EFT calculations indicates that these contributions are generally much smaller than two-body ones [22].

Nuclear matrix elements of $\mathcal{O}_{q}^{\mu_{0} \cdots \mu_{n}}$ give the moments of the isoscalar nuclear parton distributions, $q_{A}(x)$. The leading order (LO) and the next-to-leading order (NLO) contributions to these matrix elements are shown in Fig. 1(a) and (b), respectively. For an unpolarised, isoscalar nucleus,

$$
\begin{aligned}
\left\langle x^{n}\right\rangle_{q \mid A} & \equiv v^{\mu_{0}} \cdots v^{\mu_{n}}\left\langle A\left|\mathcal{O}_{q}^{\mu_{0} \cdots \mu_{n}}\right| A\right\rangle \\
& =\left\langle x^{n}\right\rangle_{q}\left[A+\left\langle A\left|\alpha_{n}\left(N^{\dagger} N\right)^{2}\right| A\right\rangle\right],
\end{aligned}
$$

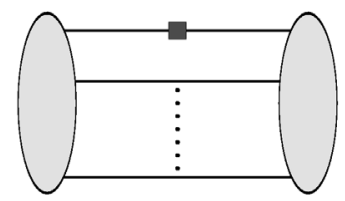

(a)

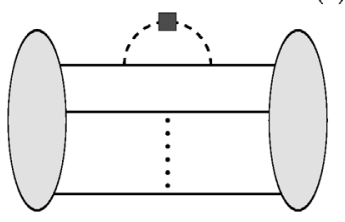

(c)

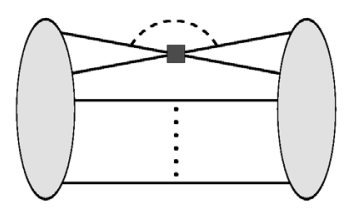

(d) where we have used $\left\langle A\left|N^{\dagger} N\right| A\right\rangle=A$. Notice that if there were no EMC effect, the $\alpha_{n}$ would vanish for all $n$. Also $\alpha_{0}=0$ because of charge conservation. Asymptotic relations [23] and analysis of experimental data [2,24] suggests that $\alpha_{1} \simeq 0$, implying that quarks carry very similar fractions of a nucleon' and a nucleus' momentum though no symmetry guarantees this. From Eq. (5) we see that the ratio

$$
\frac{\frac{\left\langle x^{n}\right\rangle_{q \mid A}}{A\left\langle x^{n}\right\rangle_{q}}-1}{\frac{\left\langle x^{m}\right\rangle_{q \mid A}}{A\left\langle x^{m}\right\rangle_{q}}-1}=\frac{\alpha_{n}}{\alpha_{m}}
$$

is independent of $A$ which has powerful consequences. In all generality, the isoscalar nuclear quark distribution can be written as

$q_{A}(x)=A[q(x)+\tilde{g}(x, A)]$.

Taking moments of Eq. (7), Eq. (6) then demands that the $x$ dependence and $A$ dependence of $\tilde{g}$ factorise,

$\tilde{g}(x, A)=g(x) \mathcal{G}(A)$,

with

$\mathcal{G}(A)=\left\langle A\left|\left(N^{\dagger} N\right)^{2}\right| A\right\rangle / A \Lambda_{0}^{3}$,

and $g(x)$ satisfying

$\alpha_{n}=\frac{1}{\Lambda_{0}^{3}\left\langle x^{n}\right\rangle_{q}} \int_{-A}^{A} d x x^{n} g(x)$.

$\Lambda_{0}$ is an arbitrary dimensionful parameter and will be chosen as $\Lambda_{0}=1 \mathrm{fm}^{-1}$. Crossing symmetry dictates

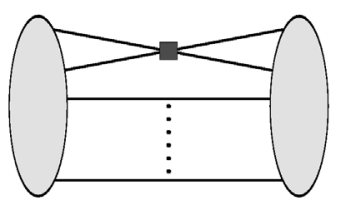

(b)

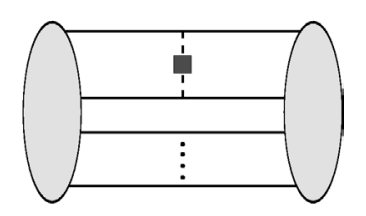

(e)

Fig. 1. Contributions to nuclear matrix elements. The dark square represents the various operators in Eq. (4) and the light shaded ellipse corresponds to the nucleus, $A$. The dots in the lower part of the diagram indicate the spectator nucleons. 
that the even and odd $\alpha_{n}$ separately determine the nuclear modifications of valence and total quark distributions. These results apply to any isoscalar combination of parton distributions including $F_{2}(x)$ for isoscalar nuclei. Thus our result implies that

$R_{A}(x)=\frac{F_{2}^{A}(x)}{A F_{2}^{N}(x)}=1+g_{F_{2}}(x) \mathcal{G}(A)$,

which says that the EMC effect (the deviation of $R_{A}(x)$ from unity) has an universal shape described by $g_{F_{2}}(x)$ while the magnitude of the deviation, $\mathcal{G}(A)$, only depends on $A$.

The above analysis gives a simple explanation of the observed universal shape of the EMC effect, or equivalently, the factorisation of $\tilde{g}(x, A)$. The key to establishing this factorisation is that other sources of nuclear modification contributing to the right-hand side of Eq. (4) must be suppressed (higher order in the EFT) such that the $A$ independence of Eq. (6) can be established. We stress that the factorisation persists when pions are included in our analysis. In Fig. 1, examples of the leading pionic contributions are shown. The various single-nucleon diagrams, such as Fig. 1(c), simply renormalise the nucleon moments, $\left\langle x^{n}\right\rangle_{q}$, without contributing to the EMC effect. Twoand more-nucleon diagrams such as those in Fig. 1(d) and (e) contribute to the EMC effect, but only at $\mathrm{N}^{3} \mathrm{LO}$ and higher (see Ref. [10] for explicit calculations) since the pions must be radiation pions rather than potential pions [25] (we are free to choose the twisttwo indices to be $\mu_{i}=0$ for all $i$ ). Other contributions that could upset the factorisation include a two-body operator which is similar to that in Eq. (4) but with two more derivatives. However this operator also contributes at $\mathrm{N}^{3} \mathrm{LO}$. Consequently, the universality of the EMC effect is preserved to good accuracy. For large $x$ it is clear that the factorisation must break down (simply consider the region $x>2$ in which only three- and higher-body operators contribute) though the structure function is very small in this region anyway. We stress that the factorisation is a model independent result and is just a consequence of scale separation and the $\mathrm{SU}(4)$ spin-isospin symmetry from large $N_{c}$.

It is clear from Eq. (9) that $\mathcal{G}(A)$ is governed by long distance physics which can be computed using a traditional, non-relativistic nuclear physics approach. It is interesting to note that the mean field scaling of $\mathcal{G}(A) \sim \rho_{A} \sim \log (A)$ describes the empirical $A$ de- pendence of $R_{A}(x)$ well [9,10], even though the mean field approximation is not justified for nuclei where the two-particle S-wave scattering lengths are much larger than the mean distance between nucleons.

Information on the shape distortion function $g(x)$ is encoded in the short distance parameters $\alpha_{n}$ associated with the strength of the two-body currents. One can either fix the $\alpha_{n}$ from experimental data (to determine all $\alpha_{n}$, data on $F_{2}^{A}(x)$ and $F_{3}^{A}(x)$ are required) or calculate $\left\langle N N\left|\mathcal{O}_{q}^{\mu_{0} \cdots \mu_{n}}\right| N N\right\rangle$ in two nucleon systems to extract them. The latter approach, however, is intrinsically non-perturbative and thus requires lattice QCD. Recent analysis [26] suggest that one can use background fields coupling to twist-two operators to extract information on the $\alpha_{n}$ from the finite volume scaling of two particle energy levels $[10,27-$ 29]. At present, only the first few coefficients could be extracted on the lattice because of problems with operator mixing [30]. However, even a calculation of these would be significant since it would be addressing nuclear modification of hadron structure from first principles. Lattice calculations can also be used to investigate the large $N_{c}$ prediction of $\beta_{n} / \alpha_{n}=O\left(1 / N_{c}^{2}\right)$ discussed below Eq. (4).

Given the success of the EFT approach in explaining aspects of the isoscalar, helicity averaged EMC effect, we shall now proceed to study the isospin and spin-dependent cases. For the isovector operators

$\mathcal{O}_{q, 3}^{\mu_{0} \cdots \mu_{n}}=\bar{q} \gamma^{\left(\mu_{0}\right.} i D^{\mu_{1}} \ldots i D^{\left.\mu_{n}\right)} \tau_{3} q /\left(2 M^{n+1}\right)$,

EFT operator matching leads to

$$
\begin{aligned}
\mathcal{O}_{q, 3}^{\mu_{0} \cdots \mu_{n}}= & \left\langle x^{n}\right\rangle_{q, 3} v^{\mu_{0}} \cdots v^{\mu_{n}} N^{\dagger} \boldsymbol{\tau}_{3} N\left[1+\alpha_{3, n} N^{\dagger} N\right] \\
& +\cdots,
\end{aligned}
$$

where the ellipsis includes the higher order nucleonic and pionic operators. Analysis similar to the isoscalar case implies

$R_{A}^{(3)}(x) \equiv \frac{q_{3 \mid A}(x)}{(Z-N) q_{3}(x)}=1+g_{3}(x) \mathcal{G}_{3}(A)$,

where $q_{3}(x)=u(x)-d(x)$ and $Z(N)$ is the proton (neutron) number. To test this factorisation, one can either consider the difference between $F_{2}$ 's in $(Z, N)=(n+m, n)$ and $(n, n+m)$ mirror nuclei [31] and compare it with $F_{2}^{p}-F_{2}^{n}$, or disentangle $u_{A}(x)$ and $d_{A}(x)$ with the proposed neutrino-nucleus experiment, MINER $v \mathrm{~A}$ [32]. 
To generalise our analysis to spin-dependent operators is also straightforward. The operators related to moments of quark helicity and transversity distributions are

$$
\begin{aligned}
& \mathcal{O}_{\Delta q, \alpha}^{\mu_{0} \cdots \mu_{n}}=\bar{q} \gamma^{\left(\mu_{0}\right.} \gamma^{5} i D^{\mu_{1}} \ldots i D^{\left.\mu_{n}\right)} \tau_{\alpha} q /\left(2 M^{n+1}\right), \\
& \mathcal{O}_{\delta q, \alpha}^{\rho \mu_{0} \cdots \mu_{n}}=\bar{q} \sigma^{\rho\left(\mu_{0}\right.} \gamma^{5} i D^{\mu_{1}} \ldots i D^{\left.\mu_{n}\right)} \tau_{\alpha} q /\left(2 M^{n+2}\right) .
\end{aligned}
$$

In the isoscalar case $\left(\tau_{0}=\mathbf{1}\right)$, the matching yields

$$
\begin{aligned}
\mathcal{O}_{\Delta q, 0}^{\mu_{0} \cdots \mu_{n}}= & 2\left\langle x^{n}\right\rangle_{\Delta q} N^{\dagger} S^{\left(\mu_{0}\right.} v^{\mu_{1}} \cdots v^{\left.\mu_{n}\right)} N \\
& \times\left[1+\gamma_{n, 0} N^{\dagger} N\right]+\cdots,
\end{aligned}
$$

and similarly for the other operators (the spin operator $S^{\mu}=(0, \sigma / 2)$ where $\sigma$ are Pauli spin matrices). Again, we have similar factorisation; e.g.,

$R_{A}^{\Delta}(x) \equiv \frac{\Delta q^{A}(x)}{\Delta N \Delta q(x)}=1+g_{\Delta}(x) \mathcal{G}_{\Delta}(A)$,

where $\Delta N$ is the differences between the number of nucleons with positive and negative spin projections in the longitudinal direction. Similarly, $\mathcal{G}_{\Delta}(A)=$ $\left\langle A\left|N^{\dagger} N N^{\dagger} \sigma_{3} N\right| A\right\rangle / \Delta N \Lambda_{0}^{3}$. Whilst there is data on longitudinal asymmetries in light nuclei from which the $g_{1}^{A}(x)$ structure functions can be extracted, disentangling nuclear effects in the unpolarised and polarised structure functions will be difficult. Currently, polarised heavy nuclei targets, in which modifications would be larger, are not available. Recent model calculations [33] find nuclear effects in the polarised structure function $g_{1}^{A}(x)$ to be significant. Nothing is known experimentally about the transversity structure function even in the proton but analogous nuclear modifications can be derived.

It is also possible to study nuclear effects in GPDs by computing off-forward matrix elements of twisttwo operators [10]. In the quark contribution to nuclear spin, $J_{q A}$, for example, in addition to extending the operators matched to $\mathcal{O}_{q}^{\mu_{0} \mu_{1}}$ in Eq. (4) by replacing $v \rightarrow v+i \frac{D}{M}$ (reparameterisation invariance [34] constrains this form), we also need to consider the term

$$
\begin{aligned}
& -2 \frac{J_{q N}}{M} i D^{\beta}\left\{\bar{N}\left[S^{\mu_{0}}, S_{\beta}\right]\left(v+i \frac{D}{M}\right)^{\mu_{1}} N\right. \\
& \left.\times\left[1+\eta N^{\dagger} N\right]\right\},
\end{aligned}
$$

where $J_{q(g) N}$ is the quark (gluon) angular momentum content of the nucleon, to obtain

$J_{q A}=\langle x\rangle_{q N} L_{z}+2 J_{q N} S_{z}[1+\eta \mathcal{H}(A)]$.

Remarkably, explicit calculation [10] shows that the $L_{z}$ term is free from two-body current corrections even though $\alpha_{1} \neq 0$ in Eq. (4). Similarly, the gluon contribution to nuclear spin satisfies

$$
J_{g A}=\langle x\rangle_{g N} L_{z}+2 J_{g N} S_{z}[1-\eta \mathcal{H}(A)],
$$

where the same constant $\eta$ appears by total angular momentum conservation. Consequently, using the sum rules $\langle x\rangle_{q N}+\langle x\rangle_{g N}=1$ and $J_{q N}+J_{g N}=1 / 2$, we recover

$J_{q A}+J_{g A}=L_{z}+S_{z}$.

For details, see [10].

To summarise, we have studied the EMC effect (nuclear modification of parton distributions) in EFT and seen that the scale separation of short and long distance effects provides a model independent derivation of the factorisation of the $x$ and $A$ dependencies of $R_{A}(x)$, relying only on the symmetries of QCD. Similar factorisations are predicted to occur in other probes of nuclear structure such as spin-dependent structure functions and GPDs.

\section{Acknowledgements}

We thank P. Bedaque, E.M. Henley, D. Lee, W. Melnitchouk, G.A. Miller, A.S. Rinat, M.J. Savage, A.W. Thomas and W.-K. Tung for useful comments and discussions. This work was supported by the US Department of Energy under contract DE-FG03-97ER41014 and the National Science Council of ROC.

\section{References}

[1] J. Aubert, et al., Phys. Lett. B 123 (1983) 275.

[2] M. Arneodo, Phys. Rep. 240 (1994) 301.

[3] D.F. Geesaman, K. Saito, A.W. Thomas, Annu. Rev. Nucl. Part. Sci. 45 (1995) 337.

[4] G. Piller, W. Weise, Phys. Rep. 330 (2000) 1.

[5] P.R. Norton, Rep. Prog. Phys. 66 (2003) 1253.

[6] J.C. Peng, et al., hep-ph/9905447.

[7] S. Date, et al., Phys. Rev. Lett. 52 (1984) 2344.

[8] L.L. Frankfurt, M.I. Strikman, Phys. Rep. 160 (1988) 235. 
[9] J. Gomez, et al., Phys. Rev. D 49 (1994) 4348.

[10] W. Detmold, J.W. Chen, in preparation.

[11] J.R. Smith, G.A. Miller, Phys. Rev. Lett. 91 (2003) 212301.

[12] See, e.g., X. Ji, J. Phys. G 24 (1998) 1181; M. Diehl, Phys. Rep. 388 (2003) 41.

[13] S.R. Beane, et al., in: M. Shifman (Ed.), Encyclopedia of Analytic QCD, World Scientific, in press; S.R. Beane, et al., Nucl. Phys. A 700 (2002) 377; P.F. Bedaque, U. van Kolck, Annu. Rev. Nucl. Part. Sci. 52 (2002) 339;

K. Kubodera, T.-S. Park, Annu. Rev. Nucl. Part. Sci. 54 (2004) 19 ;

U.G. Meissner, nucl-th/0409028.

[14] D. Arndt, M.J. Savage, Nucl. Phys. A 697 (2002) 429.

[15] J.W. Chen, X. Ji, Phys. Lett. B 523 (2001) 107; J.W. Chen, X. Ji, Phys. Lett. B 523 (2001) 73; J.W. Chen, X. Ji, Phys. Rev. Lett. 87 (2001) 152002.

[16] W. Detmold, C.-J.D. Lin, Phys. Rev. D 71 (2005) 054510.

[17] W. Detmold, et al., Phys. Rev. Lett. 87 (2001) 172001; W. Detmold, et al., Phys. Rev. D 66 (2002) 054501; W. Detmold, et al., Phys. Rev. D 68 (2003) 034025.

[18] J.W. Chen, X. Ji, Phys. Rev. Lett. 88 (2002) 052003; A.V. Belitsky, X. Ji, Phys. Lett. B 538 (2002) 289.
[19] J.W. Chen, M.J. Savage, Nucl. Phys. A 735 (2004) 441.

[20] S.R. Beane, M.J. Savage, nucl-th/0412025.

[21] D.B. Kaplan, M.J. Savage, Phys. Lett. B 365 (1996) 244.

[22] K. Kubodera, T.-S. Park, Annu. Rev. Nucl. Part. Sci. 54 (2004) 19.

[23] G.B. West, Phys. Rev. Lett. 54 (1985) 2576.

[24] A.S. Rinat, M.F. Taragin, nucl-th/0501006.

[25] D.B. Kaplan, M.J. Savage, M.B. Wise, Phys. Lett. B 424 (1998) 390;

D.B. Kaplan, M.J. Savage, M.B. Wise, Nucl. Phys. B 534 (1998) 329.

[26] W. Detmold, Phys. Rev. D 71 (2005) 054506.

[27] M. Lüscher, Commun. Math. Phys. 105 (1986) 153.

[28] S.R. Beane, et al., Phys. Lett. B 585 (2004) 106.

[29] W. Detmold, M.J. Savage, Nucl. Phys. A 743 (2004) 170.

[30] M. Göckeler, et al., Phys. Rev. D 54 (1996) 5705.

[31] K. Saito, et al., Phys. Lett. B 493 (2000) 288.

[32] D. Drakoulakos, et al., hep-ex/0405002.

[33] I.C. Cloet, et al., Phys. Rev. Lett. 95 (2005) 052302; J.R. Smith, G.A. Miller, nucl-th/0505048.

[34] M.E. Luke, A.V. Manohar, Phys. Lett. B 286 (1992) 348. 